NOTES

\title{
Molecular Weight Dependence of Tensile Properties in Poly(vinyl alcohol) Fibers
}

\author{
Hajime MrYaKe, Yasuo Gotoh, ${ }^{*}$ Yutaka OHKOSHI, ${ }^{*}$ and Masanobu NAGURA ${ }^{*}$ \\ North East Industrial Research Center of Shiga Prefecture, \\ 27-39 Mitsuyamotomachi, Nagahama-city 526-0024, Japan \\ * Faculty of Textile Science and Technology, Shinshu University, \\ 3-15-1 Tokida, Ueda 386-8567, Japan
}

(Received May 2, 2000; Accepted September 11, 2000)

KEY WORDS Poly(vinyl alcohol) / Fiber / Tensile Properties / Moisture Absorption / Molecular Weight / Molecular Motion / Molecular Chain End / Entanglement /

Poly(vinyl alcohol) (PVA) shows a high melting point of over $200^{\circ} \mathrm{C}$. Therefore, PVA is useful as an engineering material from a practical viewpoint. Kanemoto et al. attempted to prepare high tenacity and high modulus materials by drawing PVA samples of high degrees of polymerization. $^{1-3}$ Another specific property of this polymer is that it is hydrophilic due to the existence of $\mathrm{OH}$ groups. Therefore, it is important to examine the effect of moisture absorption on the physical properties of PVA fibers. It has been reported that the Young's modulus and tensile strength of a PVA fiber are lowered by absorption of moisture, whereas the tensile elongation is increased. ${ }^{4}$ However, this report did not clarify the degree of polymerization or the fine structure on the samples used. Further, there has been no report on the relation between the mechanical properties and the molecular weight of wet samples.

Recently, a method was introduced for spinning PVA with a low degree of polymerization, and such a fiber has since been marketed. ${ }^{5}$ The purpose of this study was to clarify the dependence of the fine structure of PVA fibers on the degree of polymerization and the effect of moisture absorption on their mechanical properties.

\section{EXPERIMENTAL}

\section{Materials}

Two PVA fibers with degrees of polymerization of 500 and 1750, respectively, were used (S500 and S1750; kindly supplied by KURARAY Co., Ltd.,).

\section{Tensile Properties}

Tensile properties were measured using an Instron tensile tester (Instron Japan Co., Ltd.). The tensile speed ( $5 \mathrm{~mm} \mathrm{~min}^{-1}$ ) was adjusted to minimize volatilization of absorbed water during measurements. The measurements were made immediately after removing the samples from a desiccator. Wet samples were prepared by being kept in $100 \%$ humidity in the desiccator at $20^{\circ} \mathrm{C}$ for $48 \mathrm{~h}$.

For the calculation of tensile stress, fibers were hardened with optical-setting resin (D-800; Toa Gousei Co., Ltd.) and cut perpendicular to the fiber axis with a microtome (HM360; Carl Zeiss Inc.), and then the crosssectional areas were measured using a digital micro- scope (VH6200; KEYENCE Co., Ltd.).

Young's moduli of dried samples were calculated from the gradient over strain $1 \%$ after the drawing of a waved shape of the sample itself. Young's moduli of wet samples were calculated from the initial gradient of the stress-strain curves.

\section{Moisture regain}

The moisture regain was calculated by eq 1 ,

$$
\text { Moisture regain }(\%)=\left(W_{\mathrm{w}} / W_{\mathrm{d}}-1\right) \times 100
$$

where $W_{\mathrm{w}}$ is the weight of the water-absorbed sample obtained by keeping the sample in an incubator- humidistat at $20^{\circ} \mathrm{C}$ for $48 \mathrm{~h}$ and $W_{d}$ is the weight of the dried sample after vacuum drying at $100^{\circ} \mathrm{C}$ for $24 \mathrm{~h}$.

\section{$X$-Ray Diffraction}

$\mathrm{X}$-Ray diffraction intensity curves were obtained at a scanning rate of $1^{\circ}(2 \theta) / \mathrm{min}$ and in the scanning region of $2 \theta=5-40^{\circ}$ with a diffractometer (Rigaku Denki Co., Ltd.) using $\mathrm{Cu}-K \alpha$ radiation. The crystallinity was estimated from the X-Ray diffraction intensity curves, which were measured using fibers cut in lengths of about $1 \mathrm{~mm}$ and randomly arranged. The degree of crystalline orientation on the (101) plane was calculated using the X-Ray diffraction intensity curves, which were measured while the yarn was fixed in a holder.

\section{Birefringence}

$\operatorname{Birefringence}(\Delta n)$ was calculated by eq 2 ,

$$
\Delta n=R / d
$$

where $R$ is the retardation and $d$ is the diameter of a sample. Birefringence was measured 10 times for each sample.

\section{Dynamic Loss Tangent (tan $\delta$ )}

The dynamic loss tangent was measured on a dynamic viscoelastometer (RHEOVIBRON DDV-01 FP; ORIENTIC Co., Ltd.) at a frequency of $110 \mathrm{~Hz}$ and a heating rate of $5^{\circ} \mathrm{C} \mathrm{min}^{-1}$. 
H. MiYAKE et al.

Table I. Tensile properties of PVA fibers

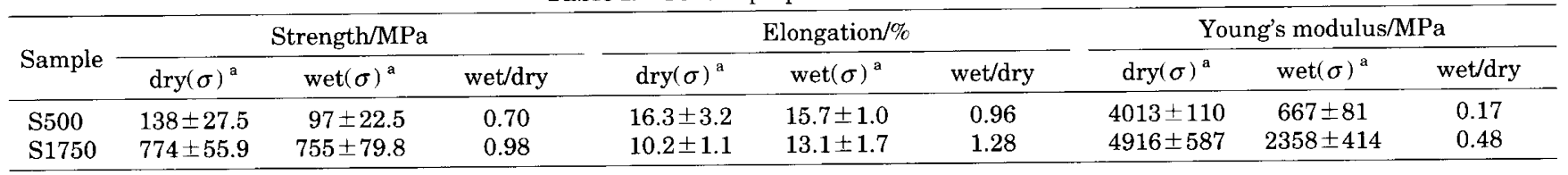

${ }^{\text {a }}$ Standard deviation.

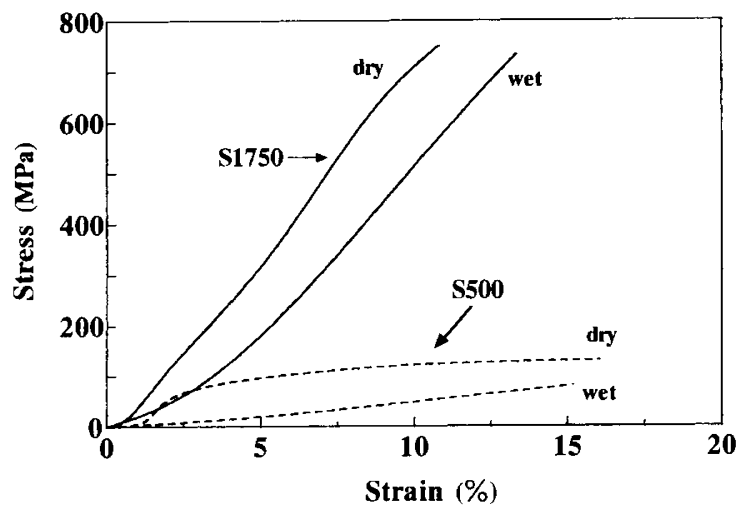

Figure 1. Stress-Strain curve of PVA fibers.

Table II. Fine structure of PVA fiber

\begin{tabular}{lcc}
\hline \multicolumn{1}{c}{ Sample } & S500 & S1750 \\
\hline Degree of crystallinity/\% & 54 & 56 \\
Degree of orientation/\% & 89 & 90 \\
Half width(X-Ray diffraction patterns) $/$ & 1.7 & 1.7 \\
\hline
\end{tabular}

\section{RESULTS AND DISCUSSION}

Figure 1 shows the stress-strain curves of the dried and the wet samples. The initial gradient of both dried samples is very small due to the wavy shape of these samples. The deformation of the dried S500 changed from elastic-type to ductility-type with elongation, while the dried S1750 showed almost no ductility deformation. The gradient of both wet samples continuously increased with elongation. And the curve of the wet S500 was comparatively gentle up to the point of rupture, while the curve gradient of the wet S1750 increased above the strain of $3 \%$.

Table I shows the Young's modulus, tensile elongation, and tensile strength of the dried and the wet samples. The Young's modulus of the dried S1750 is about double that of the dried S500. The crystallinity, crystalline orientation, and half-width of the (101) diffraction peak (namely, the crystal size) of both dried samples, obtained from X-Ray diffraction measurements, were comparable, as shown in Table II. However, the molecular mobility in the crystalline region ${ }^{6-8}$ of the dried S1750 differed from that of the dried $\mathbf{S 5 0 0}$, as shown in the temperature dependence of $\tan \delta$ (Figure 2). The $\alpha$ peak related to the molecular motion in the crystalline region appeared at around $200^{\circ} \mathrm{C}$ in the dried $\mathrm{S} 1750$ and at around $170^{\circ} \mathrm{C}$ in the dried S500. This means that the molecular mobility in the crystalline region of the dried $\mathrm{S}$ 1750 was more suppressed than that of the dried S500. In other words, there is a little of the disordered region



Figure 2. Temperature dependence of $\tan \delta$ for PVA.

formed by the absence of the intermolecular hydrogen bond in the crystalline region. ${ }^{8}$ This means that the Young's modulus of the crystalline region of the dried S1750 is higher than that of S500. This was one of the reasons for the higher Young's modulus of the dried S1750. The birefringences of the dried S1750 and S500 samples were $2.79 \times 10^{-2}$ and $3.21 \times 10^{-2}$, respectively. Since the crystallinity and the crystalline orientation of both S1750 and S500 are comparable, as mentioned above, S1750, which has a low birefringence, also has a lower degree of amorphous chain orientation than does $\mathrm{S}-500 .^{9}$ This means that the higher Young's modulus of S-1750 cannot be explained on the basis of the amorphous chain orientation. As seen in Figure 2, the magnitude of the $\beta$ peak related to the segmental motion in the amorphous region was lower in S1750 than in S500. This indicates that the amorphous chains of $\mathrm{S} 1750$ are constrained more than those of S500. Therefore, this is another cause of the higher Young's modulus of the dried S1750. S1750 had a lower degree of orientation of the amorphous chain than did S500, as mentioned above. Therefore, the smaller number of chain ends and many entanglements probably suppress the mobility of the amorphous chains in the dried S1750.

The Young's moduli of the wet samples were lower than those of the dried ones. The Young's moduli of S1750 and 5500 decreased when wetting by $52 \%$ and $83 \%$, respectively. The moisture regain of S1750 was lower than that of S500 in the whole range of relative humidity, as shown in Figure 3. This was due to the difference of the moisture regain in the amorphous region, because water molecules do not absorb in a PVA crystal. ${ }^{10}$ This indicates that the amorphous chains of S1750 are more strongly constrained in comparison with S500. Therefore, the Young's modulus of S1750 will show a small decrease with wetting. 


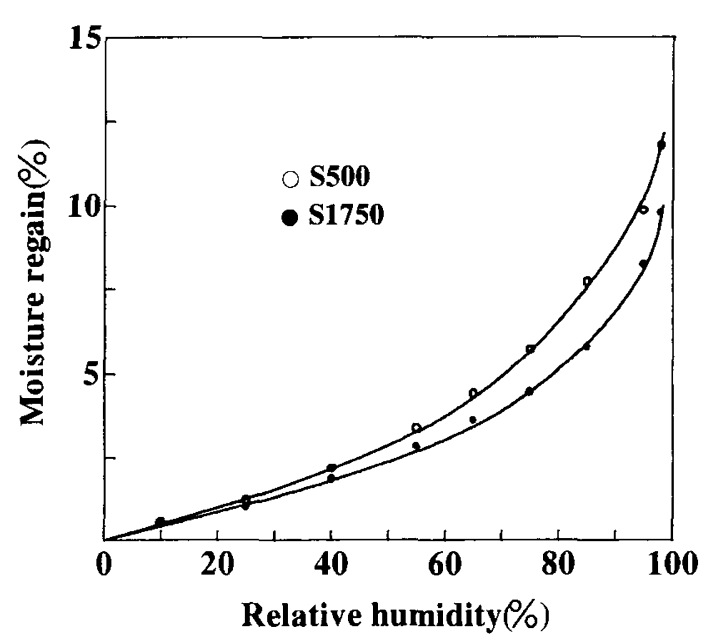

Figure 3. Isothermal sorption diagrams of PVA.

The tensile elongation of the dried S1750 is smaller than that of S500. This means that a stress concentration occurs at the short scale of plastic deformation in the former fiber. The stress concentration may occur at four possible regions:, i.e., at a defect in a crystal, at a boundary between the crystalline and amorphous regions, at the chain-end in the amorphous region, or at an entanglement point. The defect in the crystal of S1750 is

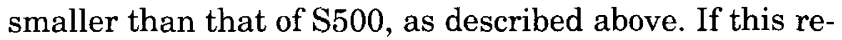
sult is correct, stress concentration in S1750 is difficult to occur, and the tensile elongation should be larger than S500. However, the opposite was the case. We therefore cannot explain the difference in tensile elongation between the two fibers by the defect in the crystal. The crystallinity was almost the same in both samples. This means that the quantity of the boundaries between the crystalline and the amorphous regions were also probably the same. Therefore, we also cannot explain the difference in tensile elongation based on the interface. Furthermore, the number of chain ends of S1750 is smaller than that of $\mathbf{S 5 0 0}$ because of the higher molecular weight of the former. This means that the stress concentration related to the chain ends may occur less readily in S1750, so that the tensile elongation of S1750 should be larger than that of S500. Here again, however, the opposite was the case. Therefore the difference in the number of chain ends cannot explain the smaller tensile elongation of S1750. Conclusively, the large number of entanglements in the amorphous region most likely causes the small tensile elongation in S1750, which act as the stress concentration points.

The birefringence of both samples decreases with wetting, as shown in Figure 4. This indicates that the initially oriented amorphous chains were slightly relaxed with wetting. The decrease of the birefringence of $\mathrm{S} 1750$ was smaller than that of the wet S500. This indicates that the amorphous chains in $\$ 1750$ relaxed less readily due to the existence of many entanglements. Further, the tensile elongation of S1750 slightly increased with

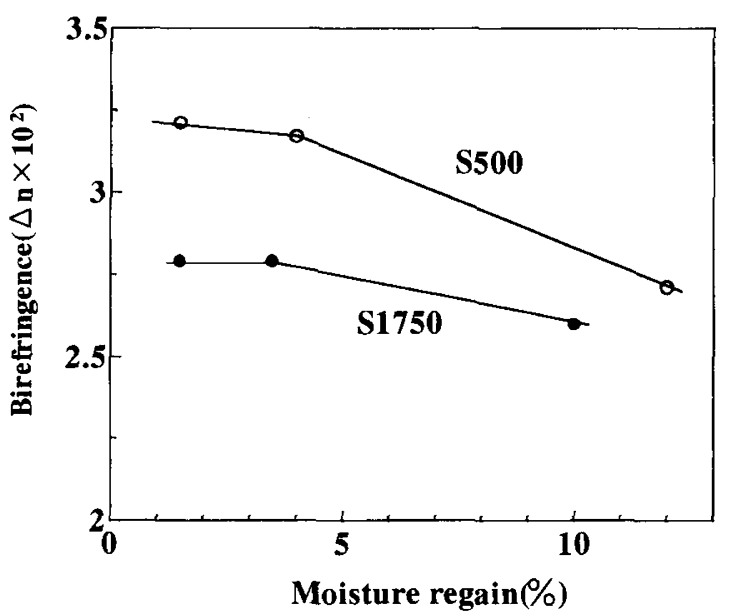

Figure 4. Dependence of birefringence on moisture regain.

wetting. This may have been due to the relaxation of the stress concentration at the entanglement points, and to the rearrangement of the amorphous chains parallel to the elongation direction during extension. In contrast, the tensile elongation of S500 decreased with wetting. This means that the amorphous chains of the wet $S 500$ can easily slip past each other due to the plasticity originated by the moisture. This was the reason for the decrease in tensile elongation by wetting in the S500 samples.

The tensile strength of S1750 did not change by wetting, whereas that of $\mathrm{S} 500$ decreased by about $30 \%$. These results were attributed to the increase in the tensile elongation, even decreasing the Young's modulus in S1750, whereas both the tensile elongation and Young's modulus decreased in S500.

Acknowledgment. The authors thank KURARAY Co., Ltd. for supplying PVA fibers.

\section{REFERENCES}

1. T. Kanamoto, A. Tsuruta, K. Tanaka, M. Takeda, and R. S. Porter, Polym. J., 15, 327 (1983).

2. T. Kanamoto, A. Tsuruta, K. Tanaka, M. Takeda, and R. S. Porter, Macromolecules, 21, 470 (1988).

3. T. Kanemoto, S. Kiyooka, Y. Tovmasyan, H. Sano, and H. Narukawa, Polymer, 31, 2039 (1990).

4. I. Sakaurada, "Polyvinyl Alcohol Fibers", Marcel Dekker Inc., New York, N.Y., $1985, \mathrm{p} 86$.

5. A. Ohmori, T. Sakuragi, and M. Onodera, Sen'i Gakkaishi, 55, 418 (1999).

6. K. Shirakashi, K. Ishikawa, and K. Miyasaka, Kobunshi Kagaku, 21, 234 (1964).

7. M. Nagura, S. Matsuzawa, K. Yamaura, and H. Ishikawa, Polym. J., 14, 69 (1982).

8. M. Nagura, S. Matsuzawa, K. Yamaura, and H. Ishikawa, Polymer Communications, 24, 250 (1983).

9. Sen’i Gakkai Hen, "Sen'i Binran", 2nd ed, Maruzen, Co., Ltd., Tokyo, 1994, p 196.

10. I. Sakurada, K. Nukusina, and Y. Sone, Kobunshi Kagaku, 12,510 (1955). 